\title{
Session 9: Anti-Infective Antibodies
}

\author{
Wednesday 9th November 2011. Moderator: Eszter Nagy
}

[09.00-09.30]

'Demonstration of antibacterial autoimmunity in acute myocardial infarction by in situ cloning of human monoclonal antibodies'

Roberto Burioni

Universita Vita-Salute San Raffaele, Milan, Italy

Abstract not provided.

\section{[09.30-09.50]}

\section{'Targeting the interferon network with synthetic} antibodies'

Shane Miersch, Ashlesha Deshpande, Srilalitha kuruganti, Kumar Putcha, Mark Walter and Sachdev Sidhu University of Toronto, Toronto, Ontario, Canada

Since the discovery of interferons (IFNs) in the late 1950's, investigation has revealed that these cytokines possesspleiotropicanti - viral, anti - tumourandimmunomodulatory activity. In light of this they have found therapeutic application in the treatment of a variety of viral, oncologic and autoimmune disorders. Alternately, dysregulation of IFNs is thought to contribute to disease in other milieus, potentially contributing to a loss of immune tolerance and the development of autoimmunity. Nevertheless, structural variations in ligand - receptor interactions that are responsible for the divergent activities that the 13+ Type I IFNs exert in binding to a single heterodimeric receptor have yet to be elucidated. Considering the uncharacterized facets of IFN biology and its potential in treating disease, antibodies that target interferons and their receptors in novel ways could aid in the characterization and modulation of receptor activation by the various isoforms. In addition to illuminating basic research questions, these antibodies would provide novel leads for the targeted and rational treatment of disease. The aim of our work is thus to develop a broad series of monoclonal antibodies to support investigation of the mechanisms of
IFN activation and to explore their potential application as novel therapeutics for the treatment of disease. Toward this objective we have employed combinatorial libraries of synthetic antibody fragments expressed on the surface of phage particles to select specifically for antibodies with these attributes and will discuss our efforts in isolating and characterizing these novel tools.

[09.50-10.10]

'Development of bispecific and multi-specific antiviral antibodies for prophylaxis and treament' Syd Johnson

Macrogenics Inc., Rockville, MA, USA

We have developed an elegant and effective platform to generate Fab-like or IgG-like bispecific antibodies. We have extended this capability to produce multispecific proteins that bind to more than two distinct antigens or epitopes. This talk will highlight bispecific and multi-specific proteins that combine subtype-specific and pan-reactive mAbs for viral prophylaxis and treatment. Respective examples for prevention/treatment of Dengue virus, Influenza virus and Smallpox will be presented.

[10.10-10.30]

'Catalytic human antibody light chains showing anti-virus activity'

Taizo Uda, Akira Nishizono and Emi Hifumi

Oita University, Oita-shi, Japan

By immunizing ground-state peptides or proteins into mice, the authors have established some catalytic antibody light chains (murine antigenases) that could destroy, (i) HIV-1 gp41, (ii) chemokine receptor CCR5 peptide (1), (iii) Helicobacter pylori urease etc (2), and (iv) TNF alpha (3). Based on their structural analysis, we proposed a concept (4), in which the antigenase en- 
codes a catalytic triad-like structure composed of Ser, His and Asp in the germline gene. It is the ultimate goal by applying the above concept to human cases to develop new therapies for patients using the advantage of human catalytic antibody.

Based on the concept, we amplified and cloned cDNAs encoding human antibody light chains belonging to subgroup II of germline genes, which were prepared from leukocytes of a volunteer vaccinated with rabies virus. As subgroup II encodes a catalytic triad-like structure with high possibility, the above cDNA (21 kinds) were transformed into E.coli and each human light chain was expressed as the protein, followed by the purification. The highly purified (over $95 \%$ ) human light chains were firstly submitted to the catalytic activity tests (amidase and nuclease) and to in vitro assays (suppression of the infectivity of viruses). Many clones including \#4 and \#18 showed the catalytic activity to cleave QAR-MCA synthetic substrate (trypsin-like) but not to cleave APA-MCA substrate (elastase-like). Surprisingly, \#4 clone exhibited a strong activity to digest pBR322 plasmid. It is noted that \#18 clone suppressed the infection of rabies virus (CVS strain) in vitro assay. In addition, we performed in vivo assays to examine whether the human antigenase affects the survival of mice. As the result, the administered mice with rabies virus treated with \#18 antigenase enhanced the number of survival mice compared with non-treated mice. In the presentation, more detail results in addition to the results of the assays against influenza virus (5) will be introduced. 